\title{
Methodology for Shape Optimization of Ultrasonic Amplifier using Genetic Algorithms and Simplex Method
}

\section{Conference Paper}

Author(s):

Deibel, Karl-Robert; Wegener, Konrad

Publication date:

2013

Permanent link:

https://doi.org/10.3929/ethz-a-009903337

Rights / license:

In Copyright - Non-Commercial Use Permitted 


\title{
METHODOLOGY FOR SHAPE OPTIMIZATION OF ULTRASONIC AMPLIFIER USING GENETIC ALGORITHMS AND SIMPLEX METHOD
}

\author{
Karl-Robert Deibel, Konrad Wegener \\ Institute of Machine Tools and Manufacturing \\ Department of Mechanical and Process Engineering \\ ETH Zurich \\ 8092 Zurich, Switzerland
}

\begin{abstract}
Designing devices for ultrasonic vibration applications is mostly done by intuitively adjusting the geometry to obtain the desired mode of vibration at a specific operating frequency. Recent studies have shown that with optimization methods, new devices with improved performance can be easily found. In this investigation, a new methodology for designing an ultrasonic amplifier through shape optimization using Genetic Algorithms and Simplex Method with specific fitness functions is presented. Displacements at specific functional areas, main functionality, and mode frequency are considered to determine the properties of an individual shape to meet the stated criteria. Length, diameter, position of mountings, and further specific geometric parameters are set up for the algorithm search for an optimized shape. Beginning with genetic algorithms, the basic shape fitting the stated requirements is found. After that the simplex method further improves the found shape to most appropriately minimize the fitness function. At the end, the fittest individual is selected as the final solution. Finally, resulting shapes are experimentally tested to show the effectiveness of the methodology.
\end{abstract}

\section{KEYWORDS}

optimization, genetic algorithm, simplex, ultrasonic, devices, amplifier

\section{INTRODUCTION}

Recent developments in ultrasonic vibration assisted machining call for new designs of tools, horns, boosters, and other components. While a wider application range of the hybrid technology of ultrasonic vibration assistance for many manufacturing technologies is of great interest, one key challenge is the design of the system in order to achieve the desired resonance vibration. The most common mode used in ultrasonic-vibrationassisted machining is the longitudinal mode of an axisymmetrical horn, which can be easily obtained when solving the Webster Horn Equation [1].

$$
\frac{\partial^{2} u}{\partial z^{2}}+\frac{1}{A(z)} \frac{d A(z)}{d z} \frac{\partial u}{\partial z}=\frac{1}{c^{2}} \frac{\partial^{2} u}{\partial t^{2}}
$$

where $t$ is time, $u$ is displacement, $A(z)$ is the cross sectional area as function of position $z$, and $c$ is the acoustic velocity. The acoustic velocity $c$ can be obtained with

$$
c=\sqrt{\frac{E_{Y}}{\rho}}
$$

where $E_{Y}$ is the material's Young's modulus, and $\rho$ the material density. In case of a harmonic motion, equation (1) can be rewritten as

$$
\frac{\partial^{2} u}{\partial z^{2}}+\frac{1}{A(z)} \frac{d A(z)}{d z} \frac{\partial u}{\partial z}=\frac{\omega^{2}}{c^{2}} u
$$

where $\omega$ is the angular frequency. Using equation (3) the length of axisymmetric horns can easily be calculated given a specific resonance frequency. The design of tool holders and horns can be obtained by solving the above equations [2-4] for various $A(z)$. 
Based on these findings, a great variety of axisymmetric horns has been found and are used in the industry today. In medical engineering, a novel ultrasonic vibration tool for surgery has been designed and tuned to the appropriate frequency for the optimal configuration [5]. A percussive drill system was designed for rock coring on planetary robotic missions using ultrasonic vibration assistance to reduce power and torque requirements [6]. More challenging are new designs for ultrasonic vibration assisted machining by combining two modes for operational purposes [7]. The longitudinal-torsional composite mode allows advanced applications for machining like drilling. Tsujino et al. designed a one-dimensional longitudinal-torsional vibration converter using diagonal slits within the resonating structure [8]. Designing transducers for ultrasonic assisted wire bonding with finite element method has been discussed in [9] with the goal of matching simulation results with experimental results. Enhancing vibration performance and matching simulation with experimental results has also been discussed in [10] for ultrasonic block horns. Properly designing a rotary ultrasonic milling tool with finite element method is introduced in [11]. For many of the mentioned designs of horns and ultrasonic vibration components, intuitive design strategies were used by evaluating the nodal displacements of modes simulated with an FEM software. A common non-automated design procedure can be found in [12], which outlines the step-by-step procedure to manually design an ultrasonic device. Automating the intuitive/manual design process can be done by structural optimization.

Many optimization methods have been applied for finding shapes that provide good results for ultrasonic vibration applications. Combining multi-objective decision making such as the NIMBUS method with the finite element method can provide very good designs as shown in [13]. Another very good optimization was introduced in [14] to find advanced transducer designs while satisfying conflicting optimal values in the design space. In [15], design of experiments is used to find the correct parameters for an ultrasonic linear motor and perform a sensitivity analysis for each parameter and their interactions with each other. Based on equation (1), a ultrasonic horn optimization method is done in [16]. Porto et al. developed a genetic algorithm to optimize the amplitude of a surgical ultrasonic transducer by changing the length of specific geometric parts at a given frequency [17].

Designing the components for ultrasonic vibration assisted machining is generally challenging, because the high frequency vibrations need to precisely occur at the tool edge or a preferred location. While the maximum amplitude is desired at the tool, minimal vibration should occur at the clamping or mounting of the ultrasonic vibration device. In this investigation, a new methodology for a shape optimization [18] of ultrasonic vibration amplifiers and reducers using generic algorithm (GA) and simplex method $[19,20]$ is introduced. Since the GA is capable of searching for the optimum of the entire design space for non convex problems, it serves as a global search method. The subsequent simplex method, as a local search method, is used to further refine the found optimum. The optimized shapes are experimentally tested by conducting an FFT Analysis and measuring displacements using a laser vibrometer.

\section{OPTIMIZATION METHODOLOGY AND PARAME- TERIZATION \\ 1.1 Methodology}

To optimize the shape of a structure via shape optimization, specific parameters need to be altered while the optimization algorithm tries to satisfy the defined criteria. Such criteria can be stress levels, compliance, volume and others that are to be maximized or minimized. As mentioned earlier, most of the ultrasonic vibration assisted machining use the longitudinal resonance mode to impose oscillations to the tool. For this optimization, the longitudinal resonance mode in $\mathrm{z}$-direction is described by the displacements of specific structural parts when exciting the structure with the operating frequency of $\omega=35 \mathrm{kHz}$ with an amplitude of $x_{0}=10 \mu \mathrm{m}$. This frequency is set by the generator (see section 2) used later in the experiments. The GA uses a fitness function that is to be minimized in order to find the optimal solution to the defined problem. For designing the structure, the finite element method (FEM) results of a modal analysis [21] and harmonic response analysis [22] are evaluated based on the displacements of the mesh nodes at the specific structure parts like cutting edge and mountings. The nodal displacement for describing the modal shape is used, because vibration modes are usually graphically evaluated (e.g. [12]) through the nodal displacements. Therefore, the global fitness function is made up from the following set of dimensionless functions. At the functional area or tool edge, the sum of displacements in z-direction is to be maximized while the sum of displacements in y-direction and the sum of displacements in $\mathrm{x}$-direction are to be minimized, as stated by

$$
f_{1}=\frac{\sum_{i=0}^{n}\left|u_{x i}\right|+\sum_{i=0}^{n}\left|u_{y i}\right|}{\sum_{i=0}^{n}\left|u_{z i}\right|} \stackrel{!}{=} \min
$$

where $u_{x}$ is the nodal displacement in X-direction, $u_{y}$ is the nodal displacement in y-direction, and $u_{z}$ is the nodal displacement in $\mathrm{z}$-direction as shown in Figure 1. With this function, bending or torsional modes will have a higher fitness value than the longitudinal mode. To ensure an even displacement across all mesh nodes at the functional area, the sum of difference between the displacement of a single node and the average displacement in 
z-direction needs to be minimized, which is stated by

$$
f_{2}=\frac{\sum_{i=0}^{n}\left(\left|u_{z i}\right|-\left|\bar{u}_{z}\right|\right)}{\left|\bar{u}_{z}\right|} \stackrel{!}{=} \min
$$

with

$$
\bar{u}_{z}=\frac{1}{n} \sum_{i=0}^{n} u_{z i}
$$

being the average displacement in z-direction. Considering the overall longitudinal mode and the placement of the mountings needed for fixing the device, it is necessary to position the mountings at the vibration nodes of the device, because minimal vibration should occur at the mountings. This is done with

$$
f_{3}=\frac{\left|x_{m 1}-x_{n 1}\right|}{\left|x_{n 1}\right|}+\frac{\left|x_{m 2}-x_{n 2}\right|}{\left|x_{n 2}\right|} \stackrel{!}{=} \min
$$

where $x_{m 1}, x_{m 2}$ are the positions of the mountings 1 and 2, and $x_{n 1}, x_{n 2}$ are the locations of the vibration nodes 1 and 2 . The values for the location of the vibration nodes are selected by finding two minima of the displacement values in z-direction along the axis of rotation. Since the input amplitude is $10 \mu \mathrm{m}$ and a booster or amplifier aims to increase the amplitude while an amplitude reducer aims to decrease the amplitude of an ultrasonic vibration system, the fitness function

$$
f_{4}=\left|A_{R}-\frac{\left|\bar{u}_{z o}\right|}{\left|\bar{u}_{z e}\right|}\right| \stackrel{!}{=} \min
$$

is formulated with $A_{R}$ being the amplitude ratio between input and output, $\bar{u}_{z o}$ is the average amplitude in z-direction at the output, and $\bar{u}_{z e}$ is the average amplitude in z-direction at the input. While a harmonic response analysis is used to obtain the results for the so far described fitness functions, a modal analysis is additionally performed to obtain the resonance frequency. Ideally, the ultrasonic device resonant frequency or mode should be equal to the operating frequency of the transducer (Section 2). This condition is stated by

$$
f_{5}=\frac{\left|\omega_{s}-\omega_{t}\right|}{\omega_{t}} \stackrel{!}{=} \min
$$

where $\omega_{s}$ is the frequency of the vibration mode and $\omega_{t}$ is the targeted frequency, which is $35 \mathrm{kHz}$ for this ultrasonic device.

\begin{tabular}{lll}
\hline Parameter & Value Range & Unit \\
\hline$Z_{13}$ & {$[20 ; 100]$} & {$[\mathrm{mm}]$} \\
$Z_{15}$ & {$[20 ; 200]$} & {$[\mathrm{mm}]$} \\
$R_{R}$ & {$[0 ; 40]$} & {$[\mathrm{mm}]$} \\
$Z_{z 1}$ & {$\left[0 ; Z_{13}\right]$} & {$[\mathrm{mm}]$} \\
$Z_{z 2}$ & {$\left[\left(Z_{13}+R_{R}\right) ; Z_{15}\right]$} & {$[\mathrm{mm}]$} \\
$R_{1}$ & {$[5 ; 16]$} & {$[\mathrm{mm}]$} \\
$R_{2}$ & {$[5 ; 16]$} & {$[\mathrm{mm}]$} \\
\hline
\end{tabular}

Table 1: Parameters defining the shape of the geometry and their value range. Mounting width and relative height are $3 \mathrm{~mm}$.

Here, equations eqs. (5), (7) and (9) are normalized to remove any dimensions.

The global fitness function for the GA optimization results in

$$
f=\sum_{i=1}^{6} R_{i} f_{i} \stackrel{!}{=} \min
$$

where $R$ is a penalty factor which can be specified for each individual fitness functions. For the following optimization, the penalty factors are set to $R_{1}=10, R_{2}=10, R_{3}=10, R_{4}=100$, and $R_{5}=10$. These factors are chosen based on relevance of each function. For the present optimization, ensuring the correct amplification $\left(f_{4}\right)$ is most important, whereas $f_{1}, f_{2}, f_{3}$ and $f_{5}$ are valid for many different amplification factors. It should be pointed out that using the same penalty factor for all functions also leads to valid results. But regarding this optimizing, the minimization of the global fitness function was found to take longer if the functions are not weighted.

\subsection{Parameterization}

The presented optimization methodology divides the structure into fixed and variable parts that make up the entity of the component. While the fixed geometric specifications may not be altered, the variable components are parameterized according to the design space available for the optimization. Figure 1 shows the ultrasonic vibration component to be optimized to meet the optimization criteria. Shown are the variable geometric parameters that are altered during the optimization process.

From Table 1 and Figure 1, it can be seen that $Z_{15}$ must always be greater than $Z_{13}+R_{R}$. Therefore, an inequality con- 


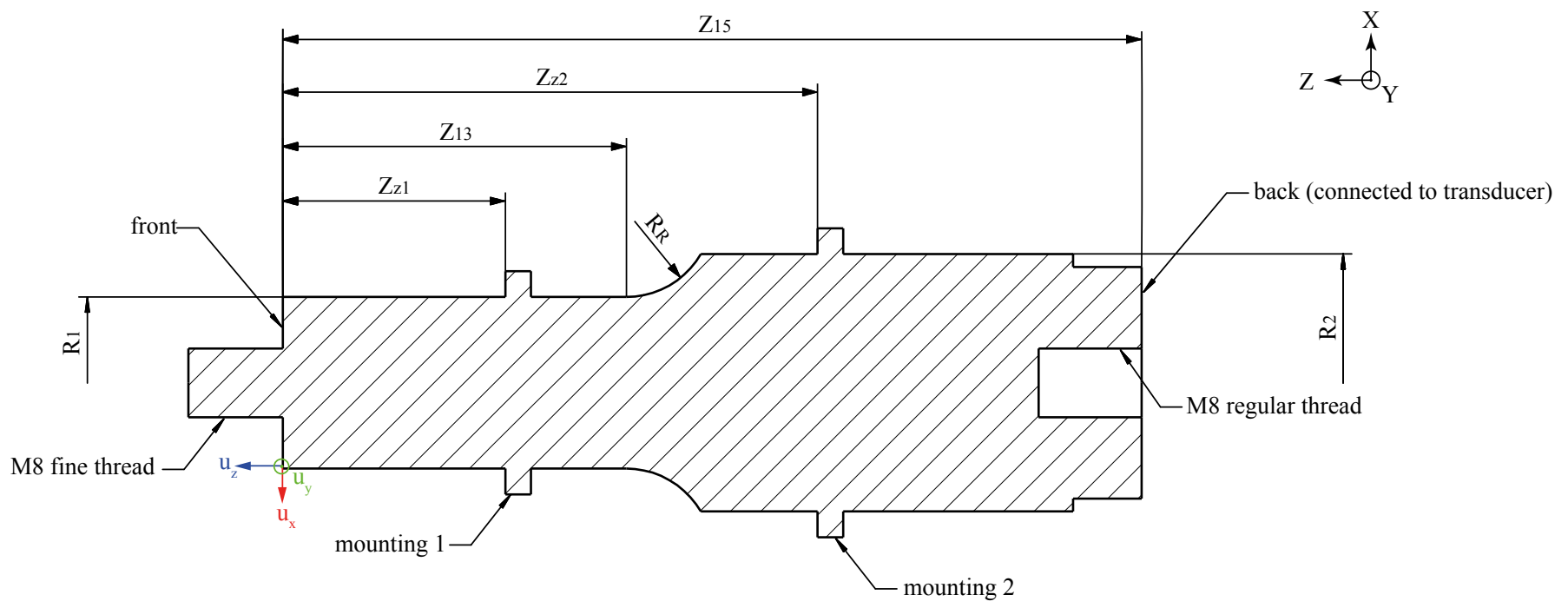

Figure 1: Cross sectional view of the geometry for optimization and the corresponding parameters. At the shown configuration, the geometry amplifies the amplitude applied to the back on the right end of the drawing. The displacements $u_{x}, u_{y}$, and $u_{z}$ for a mesh node at the front are shown.

straint needs to be defined as

$$
Z_{13}-Z_{15}+R_{R} \leq 0
$$

which the GA needs to satisfy for all solutions. Depending on how volumes are created in the FEM software, a different value can be used here instead of 0 to help with the proper geometry building. Another inequality constraint is

$$
R_{2}-R_{1}-R_{R} \leq 0
$$

to avoid invalid statements in the geometry. However, this can be replaced by simply substituting the connecting radius with a straight line for solutions with $R_{R}$ being smaller than $R_{2}-R_{1}$. While the above listed constraints should not allow invalid or unrealistic geometric shapes, any results that cause an error or invalid FEM calculation will be punished with a fitness value of infinity.

\subsection{Genetic Algorithm Settings and Simplex Method}

Figure 2 shows the essential optimization methodology. Programs used are MATLAB for the GA and ANSYS for the FEM. The GA is initialized to perform the optimization with the following settings:

- Initial Population is picked randomly within the value range (Table 1) of each parameter.

- Maximum Generation Size is 30 .
- Population Size is 500 to allow great variety among children.

- Four Elite Children will be copied from one generation to the next generation. With this, the four best solutions will be kept from one generation to the other.

- Parents are chosen using stochastic universal sampling, with more fit parents having a better chance of being picked.

- $40 \%$ of the children are obtained through mutation and the remaining $60 \%$ from crossover (reproduction).

- Mutation is done using uniform mutations at multiple points at the genotype. Mutated genes are uniformly distributed over the range defined in Table 1. If a mutant does not meet the inequality constraints, it is discarded and the mutation is repeated.

- The crossover fraction is 0.3 and reproduced children are created as the weighted arithmetic mean of two parents.

- Terminating Conditions: If the cumulative change in the fitness value does not change at least $1.0 \cdot 10^{-6}$, or the maximum of generations is reached, or no change in the fitness value (stall) is observed for 10 successive generations.

Regarding the settings of the genetic algorithm, it becomes clear that it is used with high a mutation rate to allow larger stochastic search. This is necessary, because the fitness function might have many local minima with both radii, $R_{1}$ and $R_{2}$, being within the same parameter range. Since the GA is used with high mutation, the optimization can take a longer time to find an optimum, because more individuals are used for exploring new solutions and not exploiting a known good solution. Of course, the no free lunch theorem [23] still applies for the GA. Other optimization algorithms can also be capable of solving the 


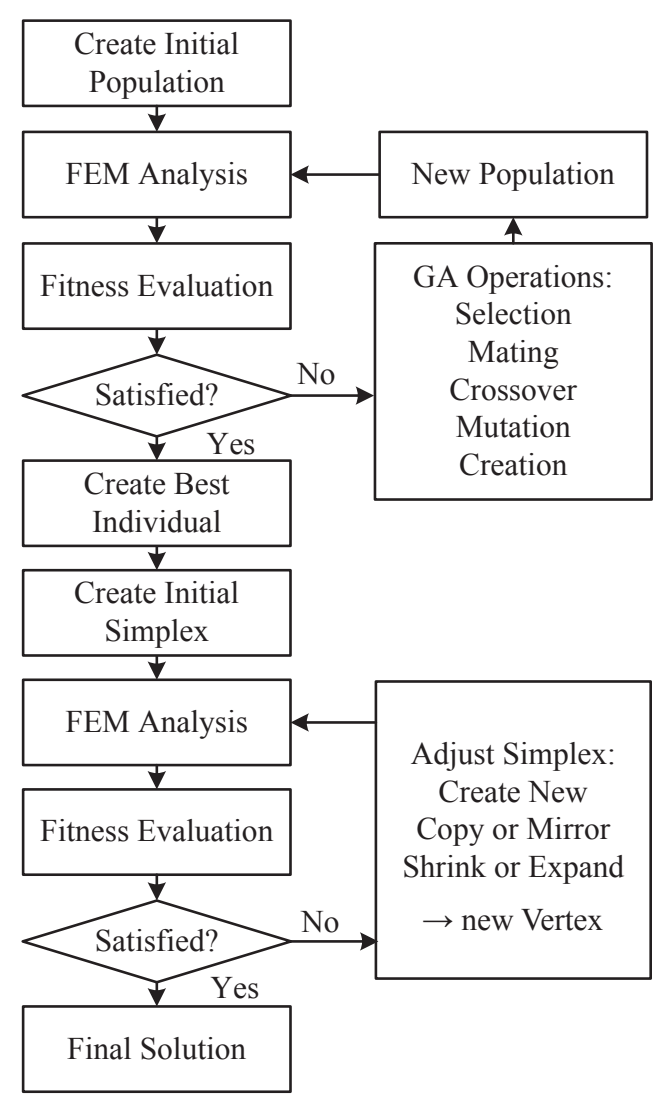

Figure 2: Optimization Methodology including GA and simplex search with described settings. FEM includes the modal and harmonic analysis. The Simplex Method picks up the best created individual of the GA and further optimizes the parameters to meet the stated criteria.

optimization problem (e.g. simulated annealing), but GA was found to be superior and faster for this specific application. The simplex method is used as a direct search method to faster optimize and refine the best individual found by the GA. This is realized by taking the optimal values found by the GA and apply the same basic methodology shown in Figure 2 and global fitness function (10) (GA operations replaced by simplex operations), except that the stopping conditions are only based on a maximum number of iterations and a relative fitness value change of $1.0 \cdot 10^{-4}$. To better reach the found minimum, the initial simplex is build with points of $5 \%$ increments found by the GA optimization, which serves as the initial vertex. Increments are slightly increased or decreased for each iteration.

\section{EXPERIMENTAL PROCEDURE AND MATERIALS}

To test the vibration amplitude and frequency of the manufactured results from the optimization, a laser vibrometer is uti-

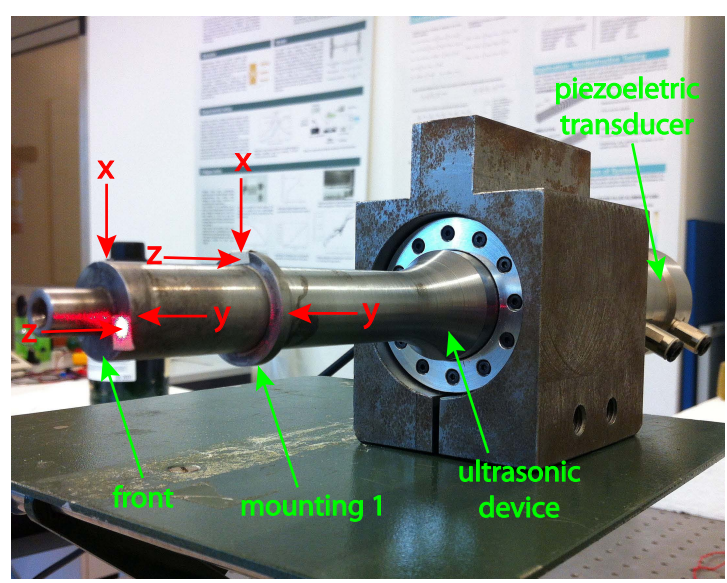

Figure 3: Measurement setup for laser vibrometer tests. Red arrows indicate the laser measurement positions.

lized. Vibrations are measured at the front and mounting 1 in $\mathrm{x}-, \mathrm{y}-$, and $\mathrm{z}$-direction. The measurement setup is shown in Figure 3 . For the measurements, mounting 2 is clamped in the holder and mounting 1 is kept free. The input amplitude is measured at the transducer output and is kept constant throughout the entire experiments. Operating power for the transducer is provided by a generator with a maximum output of $900 \mathrm{~W}$. The generator regulates the power output based on the selected amplitude setting and is designed for $35 \mathrm{kHz}$ with $\pm 500 \mathrm{~Hz}$ range. To check the frequency of the longitudinal mode of each result, a FFT analysis with $10 \mathrm{~V}$ random chirp input is done. The material used for manufacturing the ultrasonic devices is $51 \mathrm{CrV} 4$ with a Young's modulus of $E_{Y}=210 \mathrm{GPa}$, density $\rho=7850 \mathrm{~kg} \mathrm{~m}^{-3}$, Poisson ratio of 0.3 , and damping ratio of $1 \%$. Material properties used in the FEM analysis are set accordingly.

\section{RESULTS AND DISCUSSION}

To test the optimization methodology, amplifiers with amplification factors of 2 and 1 , and amplitude reducers with amplification factors of 0.5 are designed. The so far described problem and optimization procedure have multiple results that satisfy the optimization criteria. For simplification, the solutions with largest $R_{2}$ for the amplifier design and smallest $R_{2}$ for the reducer design are used for the experimental testing. Table 2 shows the values of the optimized shapes satisfying the stated criteria and minimizing the fitness function. The values of the fitness function after the GA and simplex method are also listed. It becomes very clear, that the simplex method is essential to finding an appropriate geometric shape to better minimize the stated fitness function and meet the stated criteria. Figure 4 shows the optimized shapes of the devices with amplification factors of $0.5,1$ and 2, with the mountings located at the vibration nodes of the longitudinal vibration. 


\begin{tabular}{|c|c|c|c|c|c|c|c|c|c|c|c|c|}
\hline Device & $A_{R}$ & $Z_{13}$ & $Z_{15}$ & $R_{R}$ & $\begin{array}{l}Z_{z 1} \\
{[\mathrm{~mm}]}\end{array}$ & $Z_{z 2}$ & $R_{1}$ & $R_{2}$ & Input & $\begin{array}{l}\text { Output } \\
\text { m] }\end{array}$ & $f_{G A}$ & $f_{S}$ \\
\hline \multirow{2}{*}{ Amplifier } & 2 & 83.11 & 156.66 & 40.74 & 32.12 & 118.54 & 8.88 & 15 & 10 & 17.3 & 10.03 & 7.0 \\
\hline & 1 & 62.41 & 146.69 & 39.55 & 33.75 & 105.75 & 14.8 & 15 & 10 & 9.7 & 15.5 & 2.99 \\
\hline Reducer & 0.5 & 95.81 & 145.81 & 16.86 & 32.87 & 104.95 & 12.69 & 10.79 & 10 & 7.1 & 51 & 21.6 \\
\hline
\end{tabular}

Table 2: Solutions found using the presented optimization methodology. Presented parameter values define the geometric shapes of the solutions for the tested amplification factors. Output is the average displacement at the front resulting from the FEM analysis of the final shape.

\begin{tabular}{lllllllll}
\hline \multirow{2}{*}{$A_{R}$} & \multicolumn{3}{c}{ front } & \multicolumn{6}{c}{ mounting 1} & $A_{M R}$ & $\omega_{o}$ \\
& $\hat{u}_{x}$ & $\hat{u}_{y}$ & $\hat{u}_{z}$ & $\hat{u}_{x}$ & $\hat{u}_{y}$ & $\hat{u}_{z}$ & & \\
\hline 2 & 0.02 & 0.02 & 2.39 & 0.22 & 0.25 & 0.14 & 1.9 & 35.3 \\
1 & 0.02 & 0.02 & 1.25 & 0.23 & 0.24 & 0.06 & 1.0 & 35.1 \\
0.5 & 0.02 & 0.02 & 0.72 & 0.19 & 0.19 & 0.1 & 0.6 & 35.2 \\
\hline
\end{tabular}

Table 3: Measured amplitudes at front and mounting 1 in $[\mu \mathrm{m}]$. $A_{R}$ indicates the simulated amplification factor and $A_{M R}$ states the measured amplification factor, which is calculated by dividing the measured amplitude in $\mathrm{z}$-direction at the front by the input amplitude. $\omega_{o}$ is the effective operating frequency $[\mathrm{kHz}]$ for this device.

Figure 5 shows the FFT analysis for all three devices. The operating mode at $35 \mathrm{kHz}$ can clearly be seen. Table 3 contains the measured amplitudes at the front and mounting 1 for all tested device shapes. The input amplitude for these experiments is $1.2 \mu \mathrm{m}$ at approximately $35 \mathrm{kHz}$. It can be seen that the actual amplification ratios are very near the previously stated amplification factors of the optimization. The actual output amplitudes change accordingly for each device. Vibrations measured at mounting 1 are very low indicating that it is correctly placed at the vibration node of the longitudinal mode of the device.

Regarding the solution of amplification of $A_{R}=1$, the value of $Z_{15}$ nearly matches the length of a cylindrical horn determined by solving equation (1). In case of a cylindrical horn, equation (1) can be solved to

$$
l=\frac{n \pi}{\omega} c
$$

with $l$ being the total length of the cylindrical horn, $n$ is the nth half wavelength or here the $\mathrm{n}$ number of vibration nodes, $\omega$ is the angular excitation frequency, and $c$ is the acoustic velocity [24]. Solving equation (13) with the appropriate values, the total length equals $l=147.8 \mathrm{~mm}$. It shows that the optimization procedure works for simple geometries as well. When considering function $f_{5}$, a very small change of $0.2 \mathrm{~mm}$ in length would result in a change of $100 \mathrm{~Hz}$, assuming a cylindrical horn and equation (13). Here, the simplex method is very suitable for finding the most appropriate values.

Discrepancies between simulation results and experimental results are most likely caused by inexact material properties, and numerical errors of the FEM. A change in material properties (see Section 2) affects the frequency of the vibration mode and thereby the results for the shape optimization. For example, if the Young's modulus is set as $E_{Y}=200 \mathrm{GPa}$ instead of $E_{Y}=210 \mathrm{GPa}$, the frequency goes up about $850 \mathrm{~Hz}$. It is therefore important to set the material parameters in the FEM as good as possible to the physical properties of the material used. Regarding the measured operating frequency in Table 3, the used material and geometric parameters allow a very precise FEM calculation of the solution. The element size of the mesh has an impact on the optimization as well, since with different mesh density, the results can differ.

\section{CONCLUSION}

In conclusion, it has been shown that the presented optimization methodology for ultrasonic devices is capable of delivering usable results that are in accordance with the specifications of the application. The presented fitness functions allow the optimizer to appropriately find geometric shapes of ultrasonic devices. They consist of the displacements at essential areas of the ultrasonic device and position of functional parts of the device. For finding the optimal design, a GA and simplex method is used. A longitudinal mode with the appropriate amplification at the functional area and two fix points with minimal vibration was tested for the amplitude modifiers. Changing the amplifi- 


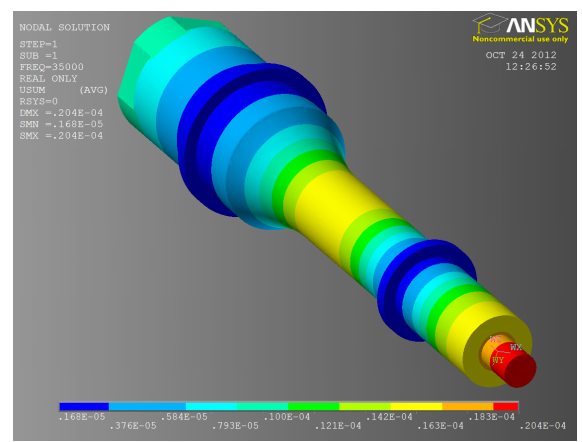

(a) Device with amplification of 2

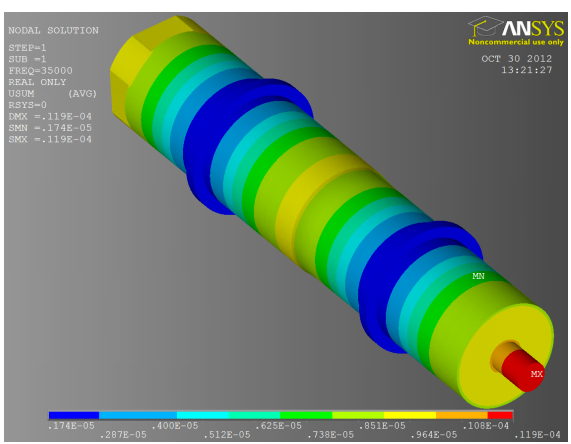

(b) Device with amplification of 1

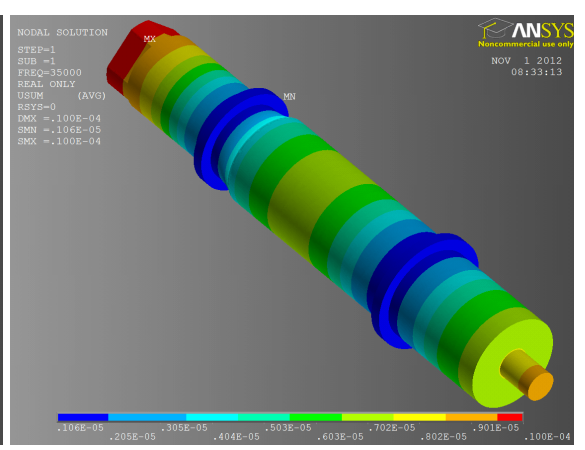

(c) Device with amplification of 0.5

Figure 4: Results of the Optimization Methodology. The longitudinal mode at $35 \mathrm{kHz}$ for all three results is shown. Colors show absolute values of displacement with an increasing amplitude at the front.

cation factor resulted in new shapes that meet the optimization criteria. Changing other specifications, such as frequency or resonance mode type, can also be done.

For further investigations, this optimization methodology needs to be tested on various ultrasonic components as well as cutting tools. To enhance the presented optimization procedure, minimization of internal stress [25] can be included in the fitness

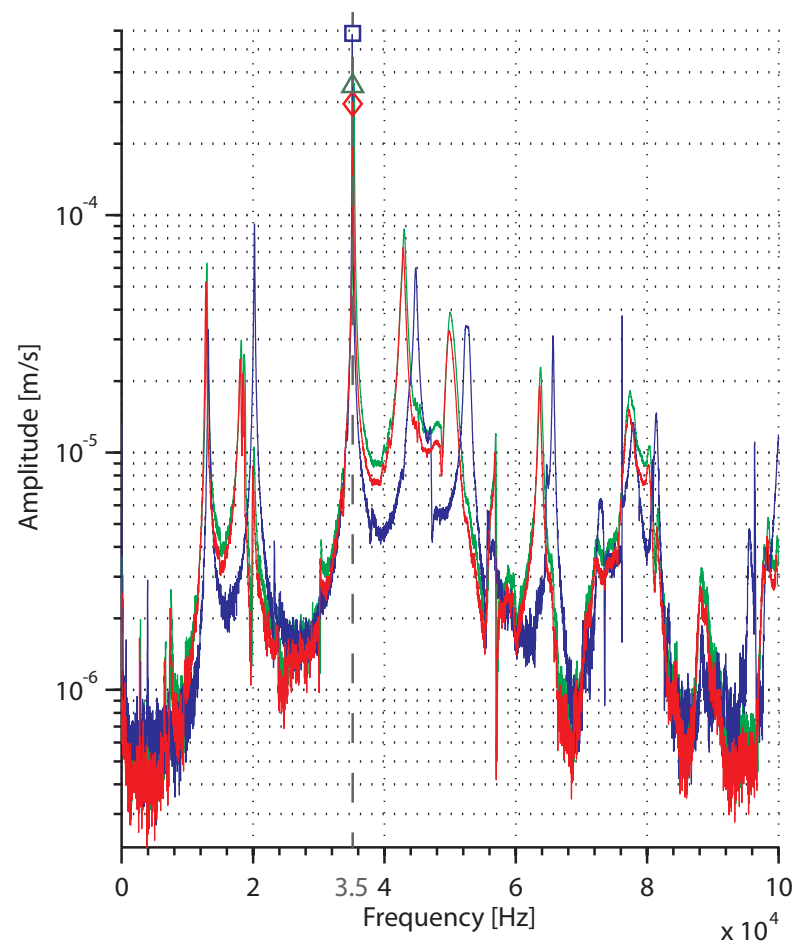

Figure 5: $\square$ shows the FFT for device with amplification 1, $\triangle$ for amplification 2, and $\diamond$ for amplification 0.5 (= reducer). Effective operating frequencies are listed in Table 3. function. This is very important for small and thin high-power ultrasonic devices. Adding further constraints to simulate the connection at the mountings might also be included. Furthermore, a topology optimization needs to be developed to enable a wider range of possible solutions for ultrasonic components. 


\section{ACKNOWLEDGEMENTS}

Special thanks go to Gerald Kress of the Institute of Mechanical Systems for the practical introduction to structural optimization, and the Institute for mechanical Systems for providing the laser vibrometer.

\section{REFERENCES}

[1] Eisner, E., 1967. "Complete Solutions of the "Webster" Horn Equation". Acoustical Society of America Journal, 41, p. 1126.

[2] Seah, K., Wong, Y., and Lee, L., 1993. "Design of tool holders for ultrasonic machining using fem". Journal of Materials Processing Technology, 37(1-4), pp. $801-816$.

[3] Amin, S., Ahmed, M., and Youssef, H., 1995. "Computeraided design of acoustic horns for ultrasonic machining using finite-element analysis". Journal of Materials Processing Technology, 55(3-4), pp. $254-260$.

[4] Sherrit, S., Askins, S. A., Gradziol, M., Dolgin, B. P., Bao, X., Chang, Z., and Bar-Cohen, Y., 2002. "Novel horn designs for ultrasonic/sonic cleaning, welding, soldering, cutting, and drilling". L. P. Davis, ed., Vol. 4701, SPIE, pp. 353-360.

[5] Qiu, Y., Huang, Z., Slade, A., and Thomson, G., 2009. “Optimization of ultrasonic tool performance in surgery". In 4th European Conference of the International Federation for Medical and Biological Engineering, Vol. 22 of IFMBE Proceedings. Springer Berlin Heidelberg, pp. 899-902.

[6] Badescu, M., Stroescu, S., Sherrit, S., Aldrich, J., Bao, X., Bar-Cohen, Y., Chang, Z., Hernandez, W., and Ibrahim, A., 2008. "Rotary hammer ultrasonic/sonic drill system". In Robotics and Automation, 2008. ICRA 2008. IEEE International Conference on, pp. $602-607$.

[7] Shuyu, and Lin, 1996. "Study on the longitudinal-torsional composite mode exponential ultrasonic horns". Ultrasonics, 34(7), pp. $757-762$.

[8] Tsujino, J., 1998. "Ultrasonic motor using a onedimensional longitudinal-torsional vibration converter with diagonal slits". Smart Materials and Structures, 7(3), p. 345.

[9] Parrini, L., 2003. "New techniques for the design of advanced ultrasonic transducers for wire bonding". Electronics Packaging Manufacturing, IEEE Transactions on, 26(1), jan., pp. $37-45$.

[10] Cardoni, A., and Lucas, M., 2002. "Enhanced vibration performance of ultrasonic block horns". Ultrasonics, 40(18), pp. 365-369.

[11] Kei-Lin, and Kuo, 2008. "Design of rotary ultrasonic milling tool using fem simulation". Journal of Materials Processing Technology, 201, pp. 48 - 52.

[12] McCulloch, E., 2008. "Experimental and finite element modelling of ultrasonic cutting of food". PhD thesis, University of Glasgow.

[13] Heikkola, E., Miettinen, K., and Nieminen, P., 2006. "Multiobjective optimization of an ultrasonic transducer using nimbus". Ultrasonics, 44(4), pp. $368-380$.

[14] Fu, B., Hemsel, T., and Wallaschek, J., 2006. "Piezoelectric transducer design via multiobjective optimization”. Ultrasonics, 44, Supplement(0), pp. e747 - e752.

[15] Fernandez, J. M., and Perriard, Y., 2006. "Sensitivity Analysis and Optimization of a Standing Wave Ultrasonic Linear Motor". IEEE Transaction on Ultrasonics, Ferroelectrics amd Frequency Control, 53(7), pp. 1352-1361.

[16] Roşca, I. C., Chiriacescu, S. T., and Creţu, N. C., 2010. "Ultrasonic horns optimization". Physics Procedia, 3(1), pp. 1033 - 1040 .

[17] Porto, D., Bourquard, A., and Perriard, Y., 2008. "Genetic algorithm optimization for a surgical ultrasonic transducer". In Ultrasonics Symposium, 2008. IUS 2008. IEEE, pp. $1457-1460$.

[18] Haslinger, J., and Mäkinen, R. A. E., 2003. Introduction to Shape Optimization: Theory, Approximation, and Computation. Society for Industrial and Applied Matematics, Philadelphia.

[19] Venkataraman, P., 2002. Applied Optimization with MAT$L A B$ Programming. John Wiley \& Sons, Inc., New York.

[20] Kress, G., and Keller, D., 2007. Structural optimization. Lecture Notes, June.

[21] He, J., and Fu, Z.-F., 2001. Modal Analysis. ButterworthHeinemann, Oxford.

[22] Petyt, M., 2010. Introduction to Finite Element Vibration Analysis, 2nd ed. Cambridge University Press, New York.

[23] Wolpert, D., and Macready, W., 1997. "No free lunch theorems for optimization". Evolutionary Computation, IEEE Transactions on, 1(1), apr, pp. $67-82$.

[24] Thomson, W. T., and Dahleh, M. D., 1998. Theory of Vibration with Applications, 5th ed. Prentice Hall, New Jersey.

[25] Cardoni, A., and Lucas, M., 2005. "Strategies for reducing stress in ultrasonic cutting systems". Strain, 41(1), pp. 1118. 